\title{
Il Traditore
}

\author{
di Davide Sparano
}

Mag 22, $2020|\underline{\text { In evidenza, }} \underline{\text { Uno sguardo su }}| \underline{0} \mid$

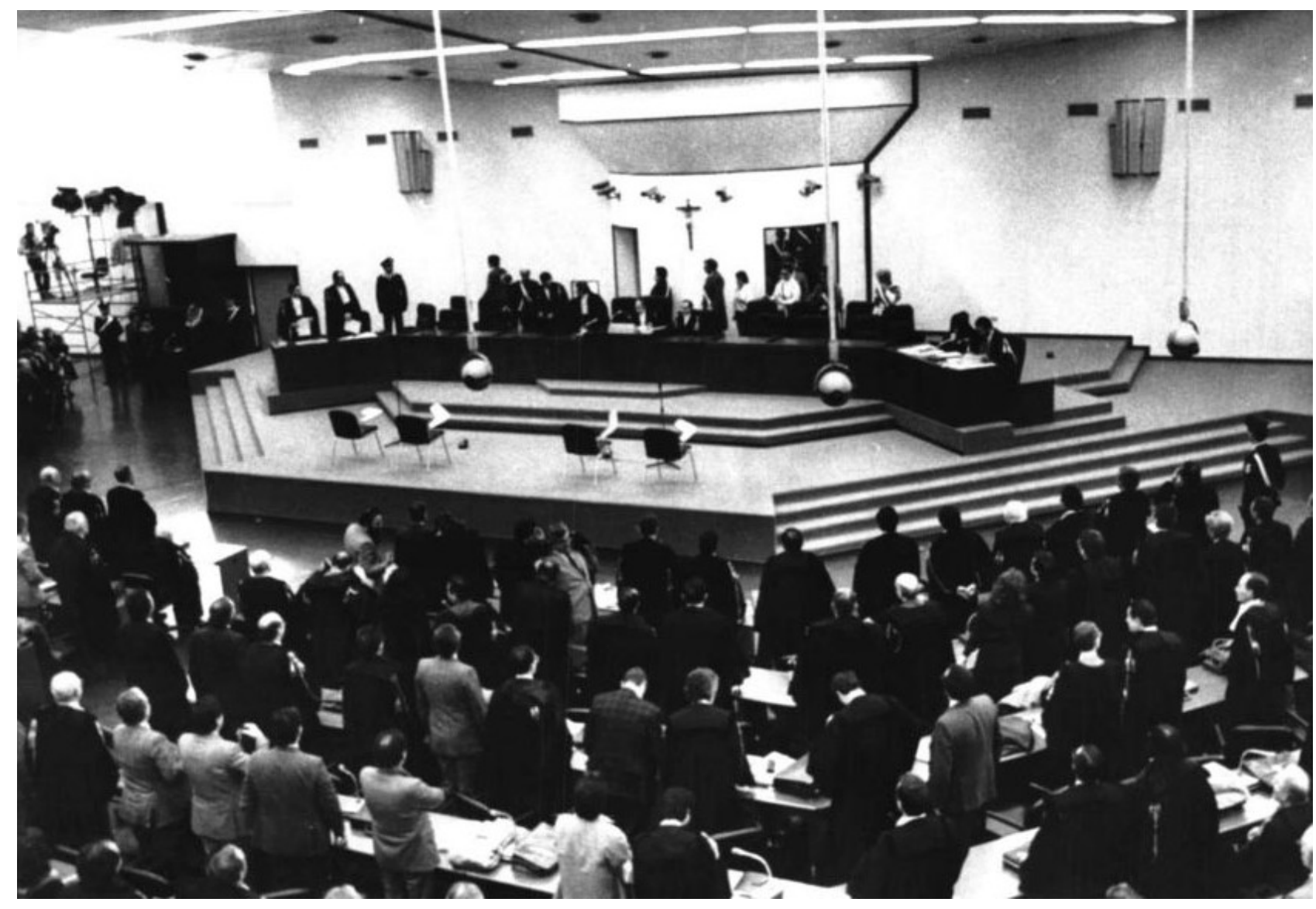

Foto del maxiprocesso di Palermo.

Di sconosciuto - sconosciuta, Pubblico dominio, Collegamento

\section{Marco Bellocchio}

\section{Il Traditore}

\section{(Italia, Francia, Germania, Brasile, 2019)}

Il Traditore, recentemente pluripremiato alla cerimonia dei David di Donatello, è un film biografico dedicato a Tommaso Buscetta, qui interpretato da un intenso Pierfrancesco Favino. La storia è quella del cosiddetto "boss dei due mondi"[1] e inizia - anche se frequenti flashback affondano in tempi molto più lontani - nei primi anni Ottanta, in una Sicilia capitale globale del traffico di droga e divisa tra le famiglie palermitane (cui Buscetta è legato) e quelle dei corleonesi di Totò Riina.

Il regista si smarca immediatamente da una narrazione preconfezionata su una vicenda notissima e recupera il Buscetta-boss dei due mondi, un uomo che ha fatto le sue fortune criminali tra l'Italia e il continente americano. Poliglotta e inserito - lo ascoltiamo parlare fluentemente portoghese Buscetta, nato da un'umile famiglia, rappresenta quella mafia che, per affari di narcotraffico, gioca e punta sul tavolo della globalizzazione. Proprio a Rio de Janeiro, dove vive ormai da anni, è tratto in arresto dalla polizia brasiliana che lo identifica e lo cattura. In seguito all'estradizione in Italia il 
boss incontra Giovanni Falcone e inizia la sua collaborazione con la giustizia: atto gravissimo per il codice d'onore mafioso e che equivale a un tradimento punibile con la morte. Quello che succederà è storia forse fin troppo nota: con le sue deposizioni Buscetta rende possibile l'istruzione del primo maxiprocesso alla mafia siciliana, tenutosi nell'aula bunker di Palermo nel 1986. La condanna in primo grado di moltissimi esponenti di spicco dell'organizzazione criminale provoca contraccolpi gravissimi, fino alla strage di Capaci del 23 maggio 1992.

Siamo di fronte ad un'opera cinematografica d'indiscusso valore nella quale emergono per lunghi tratti i fantasmi personali dell'uomo Buscetta: le dinamiche interiori, e molto complesse, di un personaggio esemplare entrato di diritto nell'immaginario collettivo,[2] s'intrecciano con un ventennio di storia italiana. L'inconscio del pentito riaffiora attraverso i sogni che sollecitano lo spettatore alla riflessione sull'ontologia del protagonista. Chi è davvero? Il semplice "soldato" di una mafia tradizionale costretta a difendersi dai sanguinari corleonesi? Oppure un furbo narcotrafficante che ha saputo proteggersi e sopravvivere?

L'opera di Bellocchio è, naturalmente, fiction, ma ci sono alcune sue caratteristiche che la rendono potenzialmente utile per spiegare certa storia del Novecento, e diverse scene possono senz'altro essere utilizzate come spunti per costruire una lezione o un percorso didattico. Come sempre, quando si tratta di un film, la scelta del docente va dalla possibilità di proporre la visione dell'opera per intero (piuttosto lunga a dire il vero: 148 minuti) o selezionare opportunamente spezzoni singoli per raggiungere precisi risultati didattici. Il film si presta a entrambe le soluzioni, soprattutto perché sembra custodire nella sua trama un'attenzione didattica e storica: date, numeri, nomi descrivono fatti, ma entrano anche nella soggettività dei personaggi e del suo protagonista. È forte la consapevolezza di trovarsi di fronte a una pellicola capace di restituire quello che Walter Benjamin definisce il «cristallo dell'evento totale»:[3] da un lato fa convivere la combinazione di elementi di passato e presente attualizzando fatti lontani nel tempo, dall'altro tenta di superare il costante schiacciamento del passato sul presente.

Non a caso il regista è molto attento al tempo che scorre: scandisce il film con una sequenza di eventi, segnalata da una vera e propria cronologia che appare sullo schermo attraverso date e didascalie. È un modo per non perdere il filo di un discorso narrativo che abbraccia vent'anni, reso con un'attenzione quasi documentaristica e richiamata graficamente con espedienti che portano alla memoria certe serie di grande successo. Immagini originali si vedono trasmesse dalla TV che manda in onda la partita Italia-Brasile dei mondiali spagnoli del 1982 e che Buscetta guarda con la sua famiglia a Rio de Janeiro. Un altro momento-chiave sono i funerali di Giovanni Falcone sintetizzati da immagini di repertorio di Paolo Borsellino che legge in chiesa e dal commovente e celeberrimo discorso della vedova Schifani.

Un ottimo esercizio di partenza per gli studenti potrebbe essere proprio quello di prendere nota di queste date per capire a cosa servono. Perché proprio queste e non altre? A quale tipo di racconto aprono? La prima è quella del 4 settembre 1980, l'ultima è il 2 aprile del 2000: in questi venti anni accade molto, ma forse i momenti più importanti sono l'incontro con Falcone (luglio 1984); il confronto con Giuseppe Calò nell'aula durante il maxiprocesso (aprile 1986); la strage di Capaci (maggio 1992). Un'altra proposta, certo più complessa, potrebbe essere quella di estrapolare dal film le numerose sezioni di impronta documentaristica per contestualizzarle e analizzarle, in una vera e propria analisi sulle fonti audiovisive.

Il 4 settembre 1980 è il giorno sul quale il film si apre: lo spettatore si trova catapultato nella splendida e fascinosa messa in scena della festa a casa del boss Stefano Bontate. Si tratta di una lunga sequenza che aiuta a tratteggiare la figura di Buscetta nella geografia dei rapporti che saranno la trama dell'intera storia. Nomi e cognomi compaiono in sovraimpressione come nella 
distribuzione di un copione teatrale, soggettività e caratteri emergono per semplificazione ma aiutano lo spettatore a entrare velocemente nella vicenda. La festa, poi, è senz'altro un omaggio e una citazione a Il Padrino di F. F. Coppola (1972), e pone il film nel solco dell'immaginario consolidato sulle mafie, nel quale le feste rituali sono il luogo di decisioni, accordi: scelte su vita e morte. Un confronto guidato fra le due sequenze potrebbe innescare un ragionamento sull'immaginario e sulla sua potenza.[4] Cosa pensano gli studenti quando si parla di mafia? Come s'immaginano la vita degli uomini e delle donne che ne sono parte? E come nascono quelle immagini? Abbiamo mai riflettuto sul fatto che la maggior parte di ciò che sappiamo - o crediamo di sapere - di queste organizzazioni criminali ci arriva dalla fiction?

Restando in tema di rappresentazione e di stereotipizzazione dei personaggi mafiosi, un altro spunto interessante è quello che nasce dal confronto tra Buscetta e Pippo Calò. I due, che in passato erano stati amici fraterni, ora sono agli antipodi e si confrontano nell'aula di giustizia. Dal loro dialogo emerge tutto il peso del linguaggio e dei codici mafiosi. Incalzato da Buscetta, Calò quasi dimentica di essere in un'aula di tribunale: un confronto tra la versione cinematografica e il filmato di quello stesso momento processuale (l'originale è reperibile su YouTube) rappresenta un interessante esercizio di comparazione fra passato/reale e presente/rappresentazione del reale.

Un'altra possibilità aperta è quella di lavorare sulla figura di Giovanni Falcone. Bellocchio sta molto attento a rappresentarlo senza che rubi la scena al protagonista: uno spettatore che abbia il ricordo preciso della storia d'Italia fra i decenni Ottanta e Novanta del secolo scorso, potrebbe persino restare spiazzato dal trovare la figura del magistrato tratteggiata senza il carisma dell'eroe civile cui ormai siamo abituati. «La mafia non esiste, la mafia è un'invenzione giornalistica. Cosa Nostra si chiama». Il dialogo tra Falcone e Buscetta inizia così: girato con una rigida successione di campi e controcampi, è il confronto tra due idee opposte di Stato e altresì molto indicativo dello sfasamento percettivo del mafioso.

Buscetta, violando la legge dell'omertà con la sua collaborazione, fornisce una chiave di lettura delle dinamiche di Cosa Nostra: «Si deve immaginare una piramide...»; da qui in classe si potrebbe partire per spiegare il funzionamento dell'organizzazione criminale.

In quella stanza i due uomini sono soli e in solitudine compiono una scelta. Le conseguenze di quella scelta sono gravissime e il film sceglie di rappresentarle entrambe: l'uccisione impietosa dei due figli di Buscetta per mano dell'amico fraterno Calò e l'attentato a Giovanni Falcone, qui rappresentato con potenza empatica e drammaticità. Il volo mortale dell'automobile in cui viaggiano il magistrato e sua moglie Francesca Morvillo lo vediamo dall'angolazione dell'autista Giuseppe Costanza, dal sedile posteriore, come fossimo lì: subito ci accorgiamo di un ribaltamento che corrisponde a uno spartiacque nella percezione pubblica della vicenda. Il 23 maggio 1992 entra nel calendario civile dando forza al complesso e lacerato tessuto della comunità nazionale. Il dolore scuote le generazioni e sfocia nei funerali di Stato trasmessi dalla televisione. Un impatto emotivo che genera la precisa percezione di una congiunzione tra «memoria pubblica e memoria autobiografica».[5]

Forse è questo il miglior tema su cui il film permette di lavorare didatticamente: ragionare con gli studenti su quale impatto abbia la storia pubblica sulle loro percezioni e sul tema del rapporto fra memoria pubblica e testimonianza privata. L'aspetto più propriamente biografico diventa la chiave per entrare nella vicenda e provare a capirla dal suo interno, seguendo passo dopo passo quella che è quasi una geo-storia della vicenda di Falcone e degli uomini che, come Buscetta, gli hanno permesso di essere quello che volevano. Le vite individuali sono i nessi che legano i singoli percorsi di vita con soggetti collettivi e istituzionali. Un passepartout che permetterebbe un ulteriore salto di 
qualità: studiare il 1992 come singolo anno chiave per la storia italiana: la fine della prima Repubblica e l'Italia nel contesto internazionale.

Buscetta muore il 2 aprile del 2000 nella sua casa in Florida, ma la sua parabola si era chiusa già nel 1996 quando aveva deciso di tornare in Italia come accusatore al processo Andreotti. È qui che il "traditore" si tradisce, incalzato dall'odioso avvocato Franco Coppi - un bravissimo Bebo Storti che ne svela l'inattendibilità come teste. È un rischio che Buscetta sapeva di correre: l'accusa è ingiusta dal punto di vista giudiziario - perché lui era un attendibile pentito - ma inevitabile dal punto di vista morale perché si trattava comunque di un trafficante, un assassino e un mafioso. Ecco la resa dei conti di Bellocchio verso il personaggio, l'abbandono del boss rimasto solo nelle mani di un avvocato che fa spietatamente il suo lavoro; proprio lui: l'implacabile assassino del padre di famiglia che rincorre per tutto il film. Il regista sgombra il campo dall'equivoco di magnificare la figura di Buscetta come esponente della "nobile mafia", ma non smette di empatizzare con lui fino alla fine quando lo ritrae cantante di karaoke nella sua versione della splendida canzone Historia de un amor (Carlos Eleta Almarán, 1955) che accompagna le scene più toccanti dell'intero film.

\section{Note:}

[1] Tommaso Buscetta aveva costruito la sua carriera criminale prima in Sicilia nella gestione del racket, nel contrabbando di sigarette e poi conquistandosi un posto importante nel traffico internazionale di stupefacenti attraverso Messico, Canada, Stati Uniti, Brasile.

[2] Sulla figura di Buscetta è incentrato il film Il Pentito (1985) di Pasquale Squitieri. Ritroviamo il personaggio anche nella serie televisiva La mafia uccide solo d'estate (2016), ideata da Pierfrancesco Diliberto (Pif), e ancora in un documentario dal titolo Our Godfather: La vera storia di Tommaso Buscetta (2019) di Mark Franketti ed Andrew Meier (andato in onda su Netflix).

[3] W. Benjamin, The Arcade Project, Belknap Press, New York, 2002.

[4] Alcuni critici sottolineano come si possano trovare riferimenti precisi anche alla scena del ballo de Il Gattopardo (Luchino Visconti, Italia, 1963); ciò che potrebbe essere stimolo a riflessioni di valore didattico interessante sulla storia dell'Italia meridionale - o meglio sulla sua rappresentazione - in una logica di più lungo periodo.

[5] M. Ravveduto, Lo spettacolo della mafia. Storia di un immaginario tra realtà e finzione, Torino, Edizioni Gruppo Abele, 2019, p. 129. 